\title{
Relationship Between Thin Cap Fibroatheroma Identified by Virtual Histology and Angioscopic Yellow Plaque in Quantitative Analysis With Colorimetry
}

\author{
Masanori Yamamoto, MD; Masamichi Takano, MD; Kentaro Okamatsu, MD; \\ Daisuke Murakami, MD; Shigenobu Inami, MD; Yong Xie, MD; Koji Seimiya, MD; \\ Takayoshi Ohba, MD; Yoshihiko Seino, MD; Kyoichi Mizuno, MD*
}

\begin{abstract}
Background: Thin cap fibroatheroma (TCFA) is considered to be a vulnerable plaque. Virtual Histology-intravascular ultrasound (VH-IVUS) can precisely identify TCFA in vivo. Intense yellow plaque on angioscopy determined by quantitative colorimetry with $\mathrm{L} * \mathrm{a} * \mathrm{~b} *$ color space corresponds with histological TCFA; in particular, a plaque of color $b^{*}$ value $>23$ indicates an atheroma with a fibrous cap thickness $<100 \mu \mathrm{m}$. In the present study, the relationship between VH-TCFA and angioscopic plaque color determined by colorimetry was investigated.

Methods and Results: Fifty-seven culprit plaques in 57 patients were evaluated by VH-IVUS and angioscopy. VH-TCFA was defined as a plaque with a necrotic core $>10 \%$ of plaque area without overlying fibrous tissue, and angioscopic TCFA was a plaque with $b^{*}$ value $>23$. The frequency of angioscopic TCFA was higher in the VH-TCFA group than in the VH-non-TCFA group (74\% vs $23 \%, \mathrm{P}=0.0002)$. Moreover, yellow color intensity (b* value) significantly correlated with plaque classification on VH-IVUS. When TCFA detected with angioscopy was used as the gold standard, the sensitivity, specificity, and accuracy for TCFA with VH-IVUS was 68\%, $81 \%$, and $75 \%$, respectively.

Conclusions: VH-TCFA strongly correlated with angioscopic TCFA determined by a quantitative analysis with colorimetry. (Circ J 2009; 73: 497-502)
\end{abstract}

Key Words: Angioscopy; Atherosclerosis; Imaging; Ultrasound

$\mathbf{T}$ he major cause of acute coronary syndrome (ACS) is disruption of an atherosclerotic plaque and the subsequent formation of a thrombus! Pathological examinations have revealed that thin cap fibroatheroma (TCFA) is frequently found in patients who die of ACS, and plaque rupture usually occurs in a lesion with an abundant necrotic core and an overlying thin fibrous cap? ${ }^{2-4}$ Recently, a spectrum analysis of intravascular ultrasound (IVUS)-derived radiofrequency data based on a pathological analysis has demonstrated more detailed quantitative information on plaque composition and morphology than a gray-scale analysis ${ }^{5-8}$ TCFA identified by Virtual Histology (VH-TCFA) are recognized more frequently in patients with ACS than in those with stable coronary artery disease.,8 Similarly, several coronary angioscopic studies have demonstrated that yellow plaques are commonly observed at the culprit lesion of ACS -13 $^{9}$ Furthermore, quantitative evaluation of the yellow plaque could be available with newly developed software using $L * a * b *$ color space! ${ }^{13-16}$ Angioscopic examination of ex vivo human tissue samples

(Received August 18, 2008; revised manuscript received October 5, 2008; accepted October 24, 2008; released online January 16, 2009) Department of Internal Medicine, Chiba-Hokusoh Hospital, Nippon Medical School, Chiba, *Division of Cardiology, Nippon Medical School, Tokyo, Japan

Mailing address: Masamichi Takano, MD, Department of Internal Medicine, Chiba-Hokusoh Hospital, Nippon Medical School, 1715 Kamakari, Imba, Chiba 270-1694, Japan. E-mail: takanom@nms.ac. jp

All rights are reserved to the Japanese Circulation Society. For permissions, please e-mail: cj@j-circ.or.jp has indicated that a coronary plaque with high-intensity, yellow color regions $\left(b^{*}\right.$ value $\left.>23\right)$ consists of lipid cores beneath a TCFA (fibrous cap thickness $<100 \mu \mathrm{m}$ ) ${ }^{15}$ However, there is no available comparison of plaque classification determined by VH-IVUS and by angioscopic findings. The present study investigated the characteristics of VH-TCFA in comparison with the angioscopic yellow color intensity as determined by quantitative colorimetry.

\section{Methods}

\section{Patient Selection}

Initially, we enrolled 67 patients who had a definable culprit plaque on pre-interventional IVUS and coronary angioscopic examinations. Ten disrupted plaques including ulceration on IVUS or massive thrombi on angioscopy (thrombus area $\geq 50 \%$ of visual field) in 10 patients were excluded because the VH analysis may have provided unreliable tissue characterization and no established algorithm for thrombus. Finally, 57 plaques in 57 patients were analyzed. The Medical Ethics Committee at Chiba-Hokusoh Hospital approved this study, and written informed consent was given by all patients before participation.

\section{Clinical Demographics}

The clinical data and information about clinical presentation were based on chart reviews. Stable angina pectoris (SAP) was defined as a positive stress test and no change in the frequency, duration or intensity of chest symptoms within 4 weeks. In each patient, the culprit lesion was identified by a combination of electrocardiographic findings, 


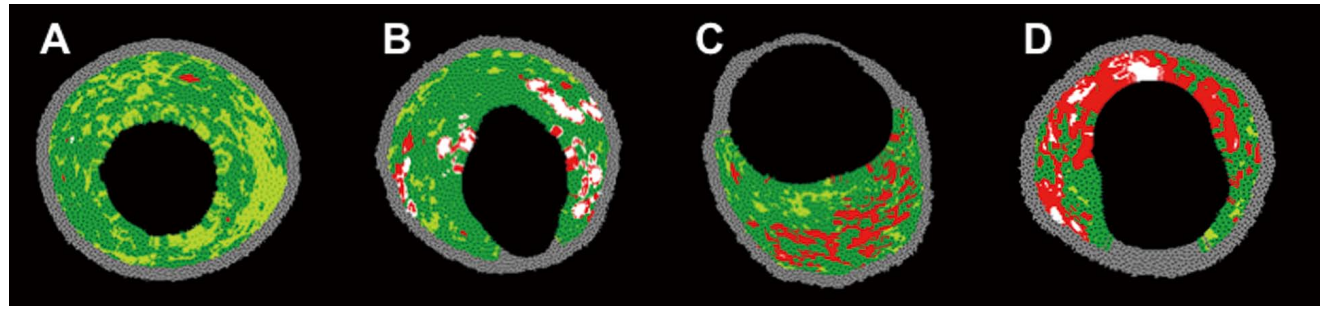

Figure 1. Plaque classification on Virtual Histology. (A) Pathological intimal thickening, composed of mainly fibrous and fibro-fatty tissue with no dense calcium and a necrotic core constituting $1 \%$ of the total plaque area. (B) Fibrocalcific lesions, featuring mainly fibrotic plaques with some dense calcium. (C) Fibrous cap atheroma: necrotic core with $18 \%$ of the total plaque area separated by fibrous tissue. (D) Thin cap fibroatheroma: superficial necrotic core rich plaque occupying $46 \%$ of the cross-sectional area without evident overlying fibrous tissue.

Table 1. Baseline Characteristics and Angiographic Findings

\begin{tabular}{lccc}
\hline \hline & VH-TCFA $(\mathrm{n}=23)$ & Non-VH-TCFA (n=34) & P value \\
\hline Age, years & $63 \pm 10$ & $61 \pm 14$ & 0.7 \\
Men & $18(78 \%)$ & $28(82 \%)$ & 0.7 \\
Diabetes & $17(70 \%)$ & $9(21 \%)$ & 0.008 \\
Hypertension & $11(48 \%)$ & $23(70 \%)$ & 0.13 \\
Hypercholesterolemia & $18(78 \%)$ & $27(79 \%)$ & 0.92 \\
Family history of coronary disease & $7(30 \%)$ & $11(32 \%)$ & 0.9 \\
Smoking & $19(83 \%)$ & $17(50 \%)$ & 0.01 \\
Observed coronary artery & $7(30 \%)$ & $8(25 \%)$ & 0.6 \\
$\quad$ Right & $11(48 \%)$ & $20(63 \%)$ & 0.3 \\
Left anterior descending & $5(22 \%)$ & $4(13 \%)$ & $<0.0001$ \\
Left circumflex & $14(61 \%)$ & $4(13 \%)$ & 0.8 \\
Acute coronary syndrome & $9(39 \%)$ & $30(87 \%)$ & 0.9 \\
Stable angina pectoris & & & 0.9 \\
Quantitative angiographic analysis & $2.7 \pm 0.6$ & $2.8 \pm 0.8$ & 0.6 \\
Reference vessel diameter, mm & $0.9 \pm 0.4$ & $0.9 \pm 0.3$ & 0.8 \\
Minimum lumen diameter, mm & $66.4 \pm 11.7$ & $66.8 \pm 11.7$ & 0.39 \\
Percent diameter stenosis, $\%$ & $11.6 \pm 4.6$ & $10.5 \pm 4.4$ & 0.8 \\
Lesion length, mm & $6(26 \%)$ & $11(33 \%)$ & $12(35 \%)$ \\
AHA/ACC classification & $11(48 \%)$ & $11(32 \%)$ & \\
Type A & $6(26 \%)$ & & \\
Type B1 & & & \\
Type B2 or C & & & \\
\hline
\end{tabular}

Values are $\mathrm{n}(\%)$ or the mean \pm SD.

VH-TCFA, thin cap fibroatheroma identified by Virtual Histology; AHA/ACC, American Heart Association/American College of Cardiology.

wall motion abnormalities on left ventriculography or echocardiography findings, and lesion morphology.

\section{Angiographic Analysis}

Quantitative angiographic measurements of the lesion were performed using digital angiograms that were analyzed with a computer-assisted, automated edge detection algorism (CMS, Medis Medical Imaging System, Nuenen, The Netherlands). The reference vessel diameter, minimum lumen diameter, percent diameter stenosis, and lesion length were measured. The lesion type was categorized according to the American Heart Association/American College of Cardiology classification.

\section{Analysis and Definition of VH-IVUS Imaging}

VH-IVUS analysis was performed during a standardized pullback $(0.5 \mathrm{~mm} / \mathrm{s})$ with the use of a VH-IVUS console and 3.5Fr imaging catheter that incorporated a $20-\mathrm{MHz}$ phasedarray transducer (Eagle Eye Gold catheter, Volcano Corp, Rancho Cordova, CA, USA). A statistical classification sorted the radiofrequency data based on combinations of the spectral parameters into 1 of 4 plaque components by color code: green (fibrous), yellow (fibro-fatty), white (dense calcium) and red (necrotic core)4-7 VH-derived plaque classifications are based on histopathological analysis ${ }^{3}$ and these criteria have been previously reported5-7 To classify the lesions, these criteria had to be met at the site of minimum lumen area plus the immediate distal and proximal crosssections. Pathological intimal thickening (PIT) was classified as a plaque composed mainly of fibrous and fibro-fatty tissue, with a lipid core or dense calcium constituting $<5 \%$ of the total plaque volume. A fibrocalcific lesion (FC) featured primarily a fibrotic plaque with dense calcium $(>5 \%$ of total plaque volume) and a lipid core occupying $<5 \%$ the total plaque volume. Fibrous atheroma (FA) was a plaque composed of fibrous and/or fibro-fatty tissue with confluent necrotic core $>10 \%$ of the total plaque volume (the necrotic core covered by a cap of fibrous and/or fibro-fatty tissue). TCFA was a necrotic core rich plaque $>10 \%$ of the crosssectional area in 3 consecutive frames without evident overlying fibrous tissue in the presence of a plaque burden $>40 \%$ (Figure 1). PIT, FC, and FA were categorized as VH-nonTCFA.

\section{Analysis and Definition of Angioscopic Imaging}

The angioscopic procedure using an imaging catheter 
Table 2. Plaque Characteristics on Virtual Histology-Intravascular Ultrasound

\begin{tabular}{lccc}
\hline \hline & VH-TCFA $(\mathrm{n}=23)$ & Non-VH-TCFA $(\mathrm{n}=34)$ & P value \\
\hline${\text { Lumen area, } \mathrm{mm}^{2}}_{\text {External elastic lumen area, } \mathrm{mm}^{2}}$ & $4.7 \pm 1.3$ & $5.6 \pm 2.0$ & 0.19 \\
Plaque area, $\mathrm{mm}^{2}$ & $15.7 \pm 5.9$ & $17.1 \pm 4.2$ & 0.51 \\
Plaque area, $\%$ & $11.5 \pm 5.5$ & $11.8 \pm 4.1$ & 0.88 \\
Fibrous, $\%$ & $67.8 \pm 8.4$ & $66.7 \pm 10.2$ & 0.78 \\
Fibro-fatty, $\%$ & $55.8 \pm 10.8$ & $67.3 \pm 9.7$ & 0.01 \\
Dense calcium, $\%$ & $10.4 \pm 5.8$ & $21.6 \pm 14.4$ & 0.02 \\
Necrotic core, $\%$ & $8.3 \pm 6.5$ & $3.7 \pm 3.9$ & 0.06 \\
Plaque with $\mathrm{b}^{*}>23$ on colorimetry & $25.5 \pm 9.5$ & $8.6 \pm 5.9$ & 0.0003 \\
\hline
\end{tabular}

Abbreviation see in Table 1.

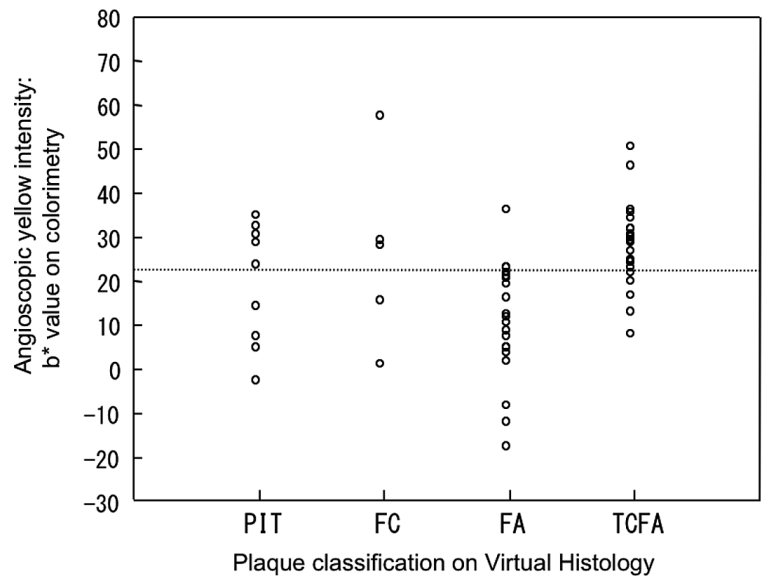

Figure 2. Relationship between plaque classification on Virtual Histology-intravascular ultrasound (VH-IVUS) and colorimetric analysis of angioscopic yellow intensity. Spearman's rank correlation analysis showed a yellow color as analyzed by quantitative colorimetry ( $\mathrm{b}^{*}$ value) to be significantly correlated with plaque classification on $\mathrm{VH}-$ IVUS ( $\mathrm{P}=0.019)$. PIT, pathological intimal thickening; $\mathrm{FC}$, fibrocalcific lesions; FA, fibrous cap atheroma; TCFA, thin cap fibroatheroma.

(Vecmova Neo, FiberTech Corp, Chiba, Japan) has been previously reported 10 The angioscopic images were digitally recorded for later analysis. The exact position of the angioscopic catheter at the observed segment was recorded on an angiogram to ensure a reliable comparison for IVUS observation. $\mathrm{L}^{*} \mathrm{a} \mathrm{b}^{*}$ color space is widely used to describe all the colors visible to the human eye (http://www.en. wikipedia.org/wiki/Lab-color-space). In this color space, a yellow color intensity can be represented as the $b^{*}$ value (yellow color intensity $0-100$, the color of blue $-100-0$ ) independent of such conditions as light intensity, the distance from the angioscope's lens to the object, and even the angle of the angioscope to the region of interest. Detailed examinations of $L * a * b *$ color space have been reported previously 13,14 and we used the same colorimetric system. Those reports revealed that a plaque of $b^{*}$ value $>23$ contains atheroma that has a fibrous cap thickness $<100 \mu \mathrm{m}$, and that such plaques are more frequently observed in the culprit lesions of ACS than in the culprit lesions of SAP. Therefore, we defined an angioscopic TCFA as a plaque with a measured $b^{*}$ value $>23$; otherwise it was deemed to be an angioscopic non-TCFA.

\section{Statistical Analysis}

Continuous variables are expressed as the mean \pm SD. Comparisons between 2 groups were performed with

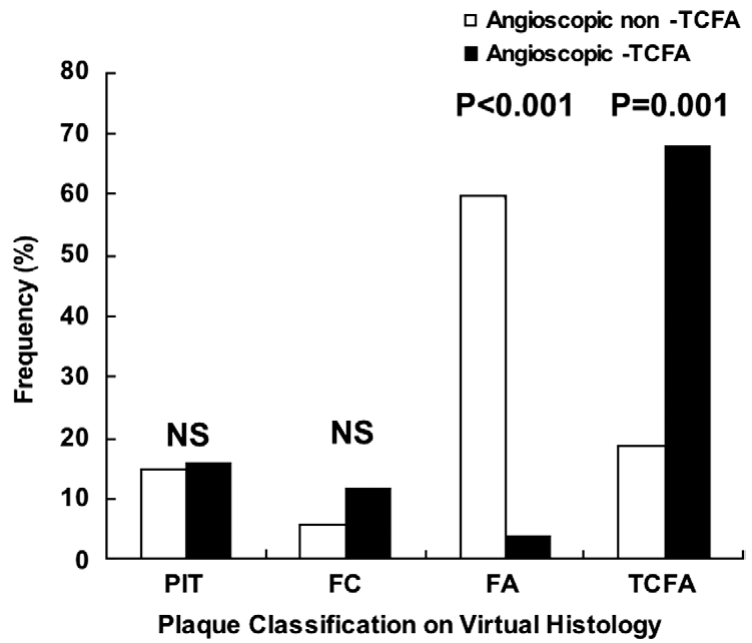

Figure 3. Distribution of angioscopic thin cap fibroatheroma (TCFA) and non-TCFA in the plaque classification on Virtual Histology (VH)intravascular ultrasound. Angioscopically analyzed plaques are divided into 25 TCFAs and 32 non-TCFAs. Of the VH-TCFA, 74\% accounted for angioscopic TCFA, while $95 \%$ of the VH-fibrous cap atheroma (FA) are angioscopic non-TCFA. The frequencies of angioscopic TCFA and angioscopic non-TCFA are similar for pathological intimal thickening (PIT) and fibrocalcific lesion (FC).

unpaired Student's t-test. Comparisons of categorical variables presented as numbers (\%) were performed by either chi-square test or Fisher's exact test. The relationship between plaque classification on VH-IVUS and angioscopic yellow color ( $b *$ value) was analyzed with Spearman's rank correlation. A P-value $<0.05$ was considered to be statistically significant.

\section{Results}

\section{Patients Characteristics and Angiographic Findings}

Baseline characteristics and angiographic findings are summarized in Table 1. There were 23 plaques classified as VH-TCFA and 34 as VH-non-TCFA. The frequencies of diabetes, smoking, and ACS were significantly higher in the VH-TCFA group (Table 1). There were no differences in the reference vessel diameter, minimum lumen diameter, percent diameter stenosis, lesion length, and type of lesion between the 2 groups.

\section{Plaque Characteristics on VH-IVUS}

Plaque characteristics on VH-IVUS are summarized in Table 2. There were no significant differences in lumen area, external elastic lumen area, plaque area, and \% plaque 

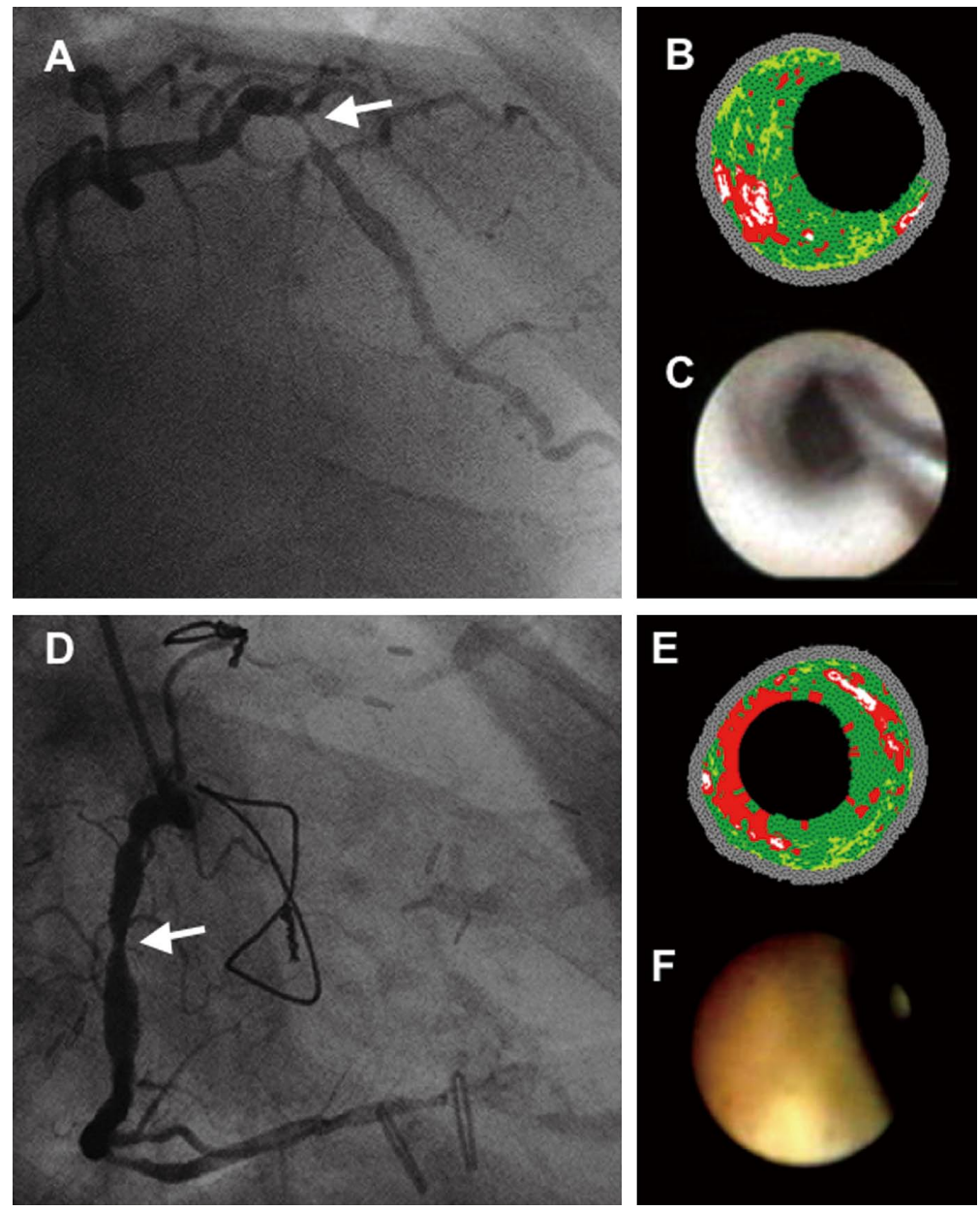

Figure 4. Corresponding Virtual Histology (VH)intravascular ultrasound (IVUS) and angioscopic images of culprit plaques. (A) Angiogram shows 90\% of stenosis (arrowhead) in the left anterior descending artery of a patient with stable angina pectoris (SAP). (B) There is no superficial necrotic core in this culprit plaque, and thick fibrous tissue covers the deep necrotic core (18\% of total plaque area) and dense calcium. This plaque is categorized as VH-fibrous cap atheroma. (C) Colorimetry indicates the yellow color intensity ( $b^{*}$ value) of this plaque to be -3.5 , and the plaque is categorized as an angioscopic non-thin cap fibroatheroma (TCFA). (D) There is 75\% stenosis in the right coronary artery of a patient with SAP (arrowhead). (E) In this culprit plaque, VH-IVUS shows a superficial large necrotic core $(41 \%$ of total plaque area) without an overlying fibrous cap. This plaque meets the criteria of VH-TCFA. (F) The yellow color intensity of this plaque is 40.6. This plaque is classified as an angioscopic TCFA. area between the 2 groups. The fibrous and fibro-fatty areas were significantly smaller in the VH-TCFA group than in VH-non-TCFA group, whereas the dense calcium and necrotic core areas were greater in the VH-TCFA group than in VH-non-TCFA group. The prevalence of angioscopic-TCFA was significantly higher in the VH-TCFA group than in the VH-non-TCFA group.

\section{Comparison of Plaque Characteristics on VH and Angioscopy}

A comparison of the $b^{*}$ value among plaque classifications on VH-IVUS is shown in Figure 2. Spearman's rank correlation analysis showed a yellow color as analyzed by quantitative colorimetry ( $\mathrm{b}^{*}$ value) to be significantly correlated with plaque classification on VH-IVUS $(\mathrm{P}=0.019)$. There were 25 angioscopic TCFAs and 32 angioscopic non-TCFAs. In the angioscopic TCFA, 17 (68\%) were classified by VH-IVUS as TCFA, 1 (4\%) was FA, 3 (12\%) were FC, and $4(16 \%)$ were PIT. Angioscopic non-TCFA were categorized as 6 TCFA (19\%), 19 FA (59\%), 2 FC $(6 \%)$, and 5 PIT (16\%) (Figure 3). VH-TCFA was more common in the angioscopic-TCFA group than in the angioscopic non-TCFA group; 17 of 23 plaques (74\%) in the VH-TCFA group accounted for angioscopic TCFA. In contrast, FA was more common in the angioscopic non-TCFA group than in the angioscopic TCFA group. In VH-FA, 19 of 20 plaques $(95 \%)$ were characterized as angioscopic non-TCFA. In plaque types PIT and FC, the frequencies of angioscopic TCFA and angioscopic non-TCFA were similar (Figure 3). Typical corresponding plaque images on VH-IVUS and angioscopy are shown in Figure 4. When TCFA detected with angioscopy was used as the gold standard, the sensitivity, specificity, positive predictive value, negative predictive value, and accuracy for TCFA with VHIVUS was $68 \%, 81 \%, 74 \%, 76 \%$, and $75 \%$, respectively.

\section{Discussion}

This study demonstrated that VH-TCFA has a more intense yellow color on the plaque surface than VH-nonTCFA. Moreover, VH-TCFA closely correlated with angioscopic TCFA, as determined by quantitative analysis with colorimetry.

Angioscopic yellow plaque is frequently observed at the culprit lesion in patients with ACS9-15 Furthermore, some prospective studies have indicated that the number of yellow plaques in the coronary arteries, or the presence of intense yellow plaques, is a predictor of future cardiac events, such as ACS 16,17 Therefore, atherosclerotic yellow plaques, especially intense yellow plaques identified by angioscopy, are believed to be vulnerable. Although angioscopic evaluation of plaque color has a major limitation because of its qualitative or semi-quantitative analysis based on visualization by the naked eye, the newly developed colorimetry enables a quantitative assessment of color with computer assistance. Comparative examination of histopathology and angioscopy findings revealed that an atheroma with fibrous cap thickness $\leq 100 \mu \mathrm{m}$ presented as an intense yellow plaque of 
$b^{*}$ value $>23$ on colorimetry ${ }^{14}$ Moreover, the typical vulnerable plaque is considered to be a TCFA with a fibrous cap thickness $<100 \mu \mathrm{m}$ ? $^{18}$ In this study, therefore, a yellow plaque of $\mathrm{b}^{*}$ value $>23$ (angioscopic TCFA) was used as the gold standard for comparison with VH-IVUS findings for tissue characterization. Coronary plaque classification using another imaging system, VH-IVUS, has been established by corresponding histopathological investigations, and this imaging modality can provide detailed information on the plaque composition in vivo ${ }^{5-8}$ The present study focused on a comparison of the identification of TCFA between VH-IVUS and angioscopy.

In this analysis of the culprit plaques, VH-IVUS identified TCFA more frequently in patients with ACS than in those with SAP. On the other hand, VH-non-TCFA was found more frequently in SAP than in ACS cases. These results are consistent with those of a previous report, 6 and the components of the culprit plaque may differ in patients with different clinical conditions. In this study, VH-TCFA was more prevalent in patients with diabetes and smoking. A previous investigation using VH-IVUS demonstrated high-frequency recognition of VH-TCFA in diabetic patients. ${ }^{19}$ Smoking induces endothelial dysfunction, thereby increasing the level of circulating oxidized low-density lipoprotein cholesterol, ${ }^{20,21}$ so not only diabetes but also cigarette smoking probably contributes to acceleration of atherosclerotic changes of coronary plaque and may contribute to the development and formation of a necrotic core.

The current results showed that a quantitative yellow intensity, the mean value of $b^{*}$ on colorimetry, significantly correlated with plaque classification on VH-IVUS. Moreover, $74 \%$ of VH-TCFA was diagnosed as angioscopic TCFA. These findings suggest that VH-TCFA closely correlates with angioscopic TCFA, and that the consistent rate of identification of TCFA was high between VH-IVUS and angioscopy. Recently, an in vivo combination study using angioscopy and optical coherence tomography (OCT) certified that the determinant factor of plaque color was not the lipid area, but the fibrous cap thickness. Most angioscopic yellow plaques were lipidic atheroma with thin fibrous caps identified by OCT, whereas atheromas with thick fibrous caps were angioscopically visualized as white plaques.2 ${ }^{22}$ It is possible that VH-TCFA, defined as plaque with a superficial necrotic core, was recognized as an intense yellow plaque.

The remaining $26 \%$ of the VH-TCFA disagreed with the angioscopic TCFA, which may be explained by several reasons. (1) Angioscopic TCFA is not always composed of a necrotic core, but rather a superficial lipid deposition, such as a fatty streak or calcified nodule! ${ }^{4}$ Although it is unclear whether a fatty streak would be categorized into the plaque type of VH-IVUS, $12 \%$ of angioscopic TCFA were actually fibro-calcific plaques on VH-IVUS. (2) According to a previous histopathological investigation, analysis based on these data showed that the predictive accuracy of $\mathrm{VH}$ IVUS for a necrotic core was limited to $85.5 \%$ in vitro and $88.3 \%$ in vivo5,23 Moreover, recent histological analysis suggested that the diagnostic accuracy of VH-IVUS was inferior in comparison with integrated backscatter IVUS 24 Therefore, VH-IVUS can not detect perfectly such a lipid plaque component, and so limitations of diagnostic accuracy still exist. (3) Although an angioscopic TCFA frequently contains lipid components, all of the lipid areas may not be recognized as necrotic core by VH-IVUS. Moreover, the necrotic core may not exceed $10 \%$ of the cross-sectional plaque area nor satisfy the criteria of $\mathrm{VH}$ -
TCFA. (4) The axial resolution of IVUS is between $100 \mu \mathrm{m}$ and $200 \mu \mathrm{m}$. In the case of an atheroma that has a fibrous cap thickness between $100 \mu \mathrm{m}$ and $200 \mu \mathrm{m}$, the atheroma may be presented as VH-TCFA because the fibrous cap overlying the necrotic core is invisible on an IVUS image. However, this type of atheroma is possibly validated as an angioscopic non-TCFA, based on determination by colorimetry. (5) In several cases, a mural thrombus on the culprit plaque may interfere with the correct plaque classification by VH-TCFA.

From the viewpoint of angioscopic diagnosis, 95\% of FA were angioscopic non-TCFA, which suggests that angioscopy thus has a limited ability to detect a profoundly lipid or necrotic core. Moreover, the distribution of angioscopic TCFA and angioscopic non-TCFA was similar for FC and PIT on VH-IVUS classification. These findings suggest that angioscopic yellow intensity is irrelevant to these plaque types.

\section{Study Limitations}

First, the comparative findings were based on observations in a relatively small number of patients. Second, 10 plaques with angioscopically massive thrombi were excluded because there is no algorithm for thrombus imaging on VH-IVUS analysis. However, this exclusion may have influenced the high consistency between VH-TCFA and angioscopic TCFA. In the future it will be necessary to develop a VH-IVUS algorithm for thrombi. Last, the images for quantitative colorimetry were obtained from stored digital-videotapes, so the image acquisition process could have affected the "absolute" $b$ * values. However, these values could be compared in all images, because they were recorded and stored in the same manner. Furthermore, b* values acquired from the digital-videotape were compared with those acquired from the previously described process without the original color correction circuit, ${ }^{15}$ using 16 standard calibration color samples (ColorChecker, X-Rite Inc, Grand Rapids, MI, USA), and there were no significant differences in the $b^{*}$ values between the 2 types of image $(\mathrm{P}=0.2$ by a paired t-test, $\mathrm{R}=0.999)$. Therefore, the "absolute" $b^{*}$ values $(=23)$ are thus considered to be acceptable in the present analysis.

Up to now, several imaging modalities to detect TCFA, such as VH-IVUS, OCT and angioscopy, have been available for clinical use. Although IVUS and angioscopy are invasive imaging techniques, analyses using VH and colorimetry enable quantitative validation of coronary plaque, and they may provide prognostic information on the natural history of high-risk plaques. Large-scale prospective studies using these imaging techniques are required to characterize true vulnerable plaques in vivo.

In conclusion, angioscopic TCFA with an intense yellow color as analyzed by quantitative colorimetry was found to be closely correlated with VH-TCFA. TCFA, a type of high-risk plaque, is likely to be identified by VH-IVUS and angioscopy with colorimetry.

\section{Acknowledgments}

The authors thank Shunsuke Shimada, Yoshihiro Abe, Toshihiro Chiba for their excellent assistance in the catheter laboratory.

\section{Disclosures}

There is no conflict of interest in this paper. Dr Xie received a research grant from Japan-Chiba Sasakawa Medical Fellowship. 


\section{References}

1. Fuster V, Badimon L, Badimon JJ, Chesebro JH. The pathogenesis of coronary artery disease and the acute coronary syndrome. $N$ Engl $J$ Med 1992; 326: 242-250, 310-318.

2. Virmani R, Burke AP, Farb A, Kolodgie FD. Pathology of the vulnerable plaque. J Am Coll Cardiol 2006; 47: C13-C18.

3. Kolodgie FD, Virmani R, Burke AP, Farb A, Weber DK, Kutyes R, et al. Pathologic assessment of the vulnerable human coronary plaque. Heart 2004; 90: 1385-1391.

4. Virmani R, Kolodgie FD, Burke AP, Farb A, Schwartz FM. Lessons from sudden coronary death a comprehensive morphological classification scheme for atherosclerotic lesions. Arterioscler Thromb Vasc Biol 2000; 20: $1262-1275$.

5. Nair A, Kuban BD, Tuzcu EM, Schoenhagen P, Nissen SE, Vince DG. Coronary plaque classification with intravascular ultrasound radiofrequency data analysis. Circulation 2002; 106: 2200-2206.

6. Rodriguez-Granillo GA, García-García HM, Mc Fadden EP, Valgimigli M, Aoki J, de Feyter P, et al. In vivo intravascular ultrasound-derived thin-cap fibroatheroma detection using ultrasound radiofrequency data analysis. J Am Coll Cardiol 2005; 46: 2038 2042.

7. Rodriguez-Granillo GA, Serruys PW, Garcia-Garcia HM, Aoki J, Valgimigli M, van Mieghem CA, et al. Coronary artery remodelling is related to plaque composition. Heart 2006; 92: 388-391.

8. Hong MK, Mintz GS, Lee CW, Suh J, Kim JH, Park DW, et al. Comparison of virtual histology of intravasucular ultrasound of culprit lesions in acute coronary syndrome and target coronary lesions in stable angina pectoris. Am J Cardiol 2007; 100: 953-959.

9. Mizuno K, Satomura K, Miyamoto A, Arakawa K, Shibuya T, Arai T, et al. Angioscopic evaluation of coronary-artery thrombi in acute coronary syndromes. $N$ Engl J Med 1992; 326: 287-291.

10. Sakai S, Mizuno K, Yokoyama S, Tanabe J, Shinada T, Seimiya K, et al. Morphologic changes in infarct-related plaque after coronary stent placement: A serial angioscopy study. J Am Coll Cardiol 2003; 42: $1558-1565$.

11. Takano M, Mizuno K, Okamatsu K, Yokoyama S, Ohba T, Sakai S. Mechanical and structural characteristics of vulnerable plaques: Analysis by coronary angioscopy and intravascular ultrasound. $\mathrm{J} \mathrm{Am}$ Coll Cardiol 2001; 38: 99-104.

12. Okamatsu K, Takano M, Sakai S, Ishibashi F, Uemura R, Takano T, et al. Elevated troponin T levels and lesion characteristics in non-STelevation acute coronary syndromes. Circulation 2004; 109: 465-470.

13. Inami S, Ishibashi F, Waxman S, Okamatsu K, Seimiya K, Takano $\mathrm{M}$, et al. Multiple yellow plaques assessed by angioscopy with quan- titative colorimetry in patients with myocardial infarction. Circ $J$ 2008; 72: 399-403.

14. Ishibashi F, Mizuno K, Kawamura A, Singh PP, Nesto RW, Waxman S. High yellow color intensity by angioscopy with quantitative colorimetry to identify high-risk features in culprit lesions of patients with acute coronary syndrome. Am J Cardiol 2007; 100: 1207-1211.

15. Ishibashi F, Yokoyama S, Miyahara K, Dabreo A, Weiss ER, Iafrati $\mathrm{M}$, et al. Quantitative colorimetry of atherosclerotic plaque using the $\mathrm{L} * \mathrm{a} * \mathrm{~b}$ color space during angioscopy for the detection of lipid cores underneath thin fibrous cap. Int J Cardiovasc Imaging 2007; 23: 679-691.

16. Uchida Y, Nakamura F, Tomaru T, Morita T, Oshima T, Sasaki T, et al. Prediction of acute coronary syndromes by percutaneous coronary angioscopy inpatients with stable angina. Am Heart J 1995; 130: 195-203.

17. Ohotani T, Ueda Y, Mizote I, Oyabu J, Okada K, Hirayama A, et al. Number of yellow plaque detected in acolonary artery is associated with future risk of acute coronary syndrome. J Am Coll Cardiol 2006; 47: $2194-2200$.

18. Naghavi M, Libby P, Falk E, Casscells SW, Litovsky S, Rumberger J, et al. From vulnerable plaque to vulnerable patient: A call for new definitions and risk assessment strategies: Part II. Circulation 2003; 108: $1664-1672$.

19. Nasu K, Tsuchikane E, Katoh O, Fujita H, Surmely JF, Ehara M, et al. Plaque characterization by virtual histology intravascular ultrasound analysis in type 2 diabetic patients. Heart 2008; 94: 429-433.

20. Vita JA, Treasure CB, Nabel EG, McLenachan JM, Fish RD, Yeung $\mathrm{AC}$, et al. Coronary vasomotor response to acetylcoline relates to risk factors for coronary artery disease. Circulation 1990; 81: 1491-1497.

21. Heitzer T, Ylä-Herttula S, Luoma J, Kurz S, Münzel T, Just H, et al. Cigarette smoking patients endothelial dysfunction of forearm resistance vessels in patients with hypercholesterolemia: Role of oxidized LDL. Circulation 1996; 93: 1346-1353.

22. Takano M, Jang IK, Inami S, Yamamoto M, Murakami D, Okamatsu $\mathrm{K}$, et al. In-vivo comparison of optical coherence tomography and angioscopy for the evaluation of coronary plaque characteristics. $\mathrm{Am}$ J Cardiol 2008; 102: $27-31$.

23. Nasu K, Tsuchikane E, Katoh O, Vince DG, Margolis PM, Virmani $\mathrm{R}$, et al. Accuracy of in vivo coronary plaque morphology assessment a validation study of in vivo virtual histology compared with in vivo histopathology. J Am Coll Cardiol 2006; 47: 2405-2412.

24. Okubo M, Kawasaki M, Ishihara Y, Takeyama U, Yasuda S, Kubota $\mathrm{T}$, et al. Tissue characterization of coronary plaques: Comparison of integrated backscatter intravascular ultrasound with virtual histology intravascular ultrasound. Circ J 2008; 72: 1631-1639. 